\title{
Improvement of the working bodies of the harvesting machines by means of the use of composite materials
}

\author{
Nikita Zhbanov, Nikolay Byshow, Natalia Kostenko, George Rembalovich, and Mihail Kostenko* \\ Ryazan State Agrotechnological University named after P.A. Kostychev, 390044 Ryazan, Russia
}

\begin{abstract}
Potato production is associated with high energy and labor costs, and the final part (harvesting) accounts for the bulk of the costs. When designing potato harvesting technology, the main focus is on the separation of soil impurities, which make up about $97 \%$ with a high yield of $300 \mathrm{~kg} / \mathrm{ha}$, and lower yields increase them to 98-99\%. Nowadays, with the advent of new materials and new technologies in agricultural engineering, there is modernization of existing structures using innovative materials, as a result of which some new and having no analogues models are created that can improve the quality of the machine, increase its service life, and reduce energy costs. The effectiveness of the working bodies of potato harvesters determines the ratio of the separating ability of the working body to its energy consumption. As a result of the studies, power parameters and the separating ability of the main working bodies were generalized. The modernization of the separating elevator consists in replacing the metal rods of an existing elevator apron with a similar apron with rods of some composite material. This modernization can significantly reduce the mass of the working body and the whole combine as a whole and reduce energy consumption.
\end{abstract}

\section{Introduction}

Potato production is associated with high energy and labor costs and harvesting accounts for the bulk of the costs. Harvesting potatoes is currently impossible to imagine without the use of harvesters that increase productivity and reduce crop losses. Modern potato harvesters are complex engineering systems that perform the following technological operations: digging up potato beds, separating soil and plant impurities, cleaning tubers and removing lumps and stones, accumulating and loading the finished product into vehicles [1, 2].

When designing potato harvesting technology, the main focus is on the separation of soil impurities, which are about $97 \%$ in a case of a high yield of $300 \mathrm{~kg} / \mathrm{ha}$, and when lower yields soil impurities increase to 98...99\% [3, 4].

The question of the reliability of potato harvesters is of no less importance. To prevent equipment failure, safety devices are widely used that can significantly increase the life of the combine and avoid failures. In addition, at present, with the advent of new materials and new technologies in agricultural engineering, there is some modernization of existing structures using innovative materials, as a result of which new and having no analogues models are created that can improve the quality of the machine, increase the service life and reduce energy consumption [5].

Based on the aforementioned, it is relevant to establish the most power-loaded areas of working bodies on a potato harvester with the subsequent development of schemes for the modernization of the most loaded working bodies. The main concept for the modernization of the working bodies will be to ensure the most effective screening separation with minimal damage to tubers by working bodies and to separate impurities at a given optimal technological mode of working bodies.

\section{Materials and methods}

The efficiency of the working bodies of potato harvesters determines the ratio of the separating ability of the working body to its energy consumption. To evaluate the working organs of the potato harvester, their separating abilities and load intensities were examined.

As a result of the studies, power parameters and the separating ability of the main working bodies were generalized. The data obtained show that the modernization of the main elevator to reduce idle energy costs is an urgent task.

One of the possible directions to modernize the working body is the replacement of an existing elevator apron with metal bars with a similar one with bars made of some composite material. This modernization can significantly reduce the mass of the working body and the harvester as a whole, as a result of which to reduce energy consumption, improve the resistance to the loads on the elevator apron, which in turn will increase the durability of this bar elevator.

The proposed model is a bar elevator of a rootharvesting machine, including a frame with a rod apron

* Corresponding author: kostenko.mihail2016@yandex.ru 
having under it some leading, driven, supporting rollers and shakers, alternating in length and width and mismatching of the phases of their raising and lowering. The model is characterized by rods made of some flexible composite material, and the shakers are rubberized rotating rollers located with the formation of a wavy surface.

Let us dwell in more detail on the design of a separating elevator with rods made of some composite material.

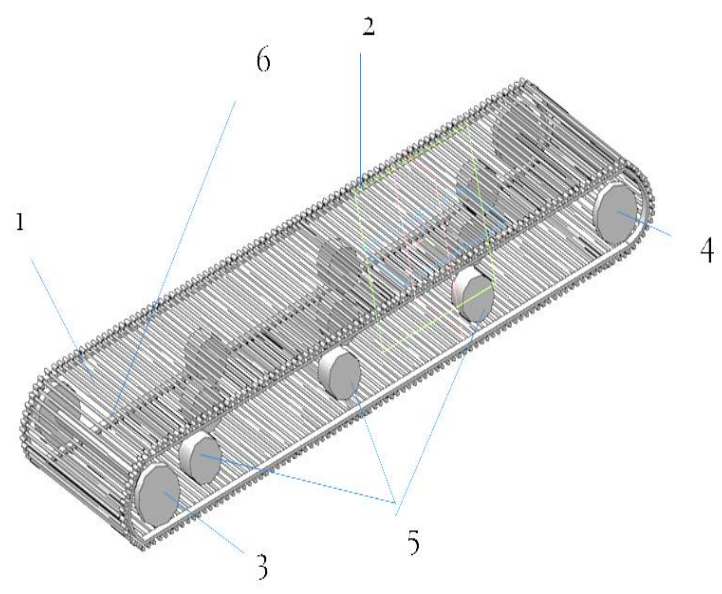

Fig. 1. Raddle chain with rods of composite material

The cleaning elevator of the root harvester machine consists of flexible traction elements 1 with combined rods, which are rods 2 with tubes 3 put on them. Driving rollers 4, supporting rollers 5 and driven rollers 6 are mounted on the elevator frame and provide web tension. Shaker 7 in the form of spirally curved rods connected to the drive is mounted under the combined rods of the elevator. Tube 3 of the combined rod is always in contact with two spiral curved rods.

Consider the mode of the device. The tuber heap comes to a rod-shaped apron with flexible composite rods. As the tuber heap moves along the raddle chain, the flexible composite elevator bars rise on the support rollers and shakers, and the bars bend under the influence of the tuber heap gravity. With further movement, a change in the surface configuration of the rod-shaped apron happens, resulting in accelerations affecting the tuber heap. When the shaker of the rodshaped apron is working, flexible composite rods are deflected, which, on the one hand, reduces the intensity of the impact, and, on the other hand, provides a large amplitude of tossing the tuber heap. The use of flexible composite rods makes it possible to reduce shock loads when the tuber heap falling and to reduce the tubers' damage [6-8].

\section{Results}

When intensifier rollers bend around the elevator apron, gravity and centrifugal gravity and centrifugal forces will act on the tuber heap particle, since the elevator apron moves at a constant speed, and the value of centrifugal acceleration will be equal to

$$
a_{c f}=\frac{V^{2}}{r}=r \frac{\pi^{2} n^{2}}{900}
$$

where $V$ is the elevator apron speed, $\mathrm{m} / \mathrm{s}$;

$r$ is intensifier roller radius, $\mathrm{m}$;

$n$ is frequency of rotation of the roller, $\mathrm{r} / \mathrm{m}$.

The elevator apron, moving with potato heaps along the intensifier rollers, has some certain flexibility of belts and rods, which leads to the bending of the surfaces of the rollers. The point of contact will depend on the speed of the elevator, the diameter of the roller, the mass of the potato heap and the parameters of the apron. The point of contact and apron flow from the roller was determined experimentally.

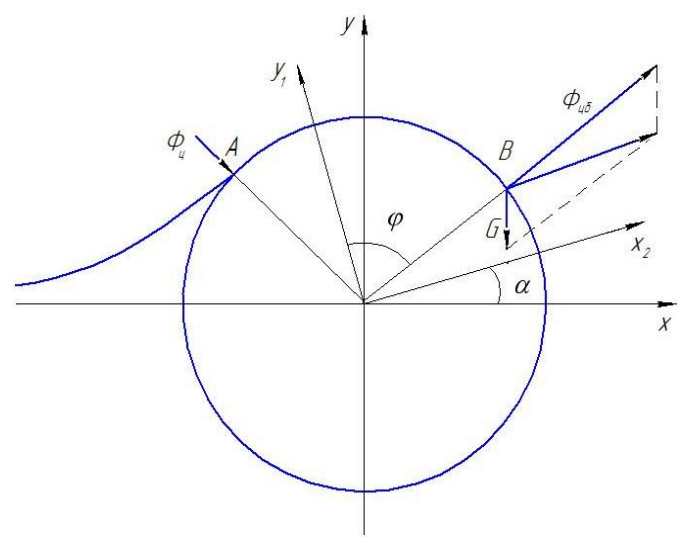

Fig. 2. Design scheme and the determination of the flow point of the potato component from the intensifier

In order to determine the moment when the components of the potato heap come off the apron, the D'Alamber principle is used and equilibrium equations are drawn up taking into account the inertia forces

$$
\vec{\Phi}_{c i}+\vec{G}=0
$$

Projecting expressions 5 on coordinate axis $x_{l} O y_{l}$ and substituting the values of forces one gets

$$
\left\{\begin{array}{l}
m a_{c f} \cos \varphi-m g \cos \alpha=0 \\
m a_{c f} \sin \alpha-m g \sin \alpha=0
\end{array}\right.
$$

where $m$ is the mass of the final component, $\mathrm{kg}$; $\alpha$ is the elevator tilt angle to the horizon; $\varphi$ is the component separation angle from the roller; $g$ is gravity acceleration, $\mathrm{m} / \mathrm{s}^{2}$.

Substitute the value of acceleration

$$
\left\{\begin{array}{l}
m \cdot \frac{V^{2}}{r} \cdot \cos \varphi-m g \cos \alpha=0 \\
m \cdot \frac{V^{2}}{r} \cdot \sin \varphi-m g \sin \alpha=0
\end{array}\right.
$$

Since the raddle chain moves at a constant speed, the value of radius rof the roller and angle $\varphi$ at which the heap component separates from the elevator apron are determined

$$
\begin{gathered}
r=\frac{V^{2} \cos \varphi}{g \cos \alpha} \\
\varphi=\arcsin \left(\frac{r \cdot g \cdot \sin \alpha}{V^{2}}\right)
\end{gathered}
$$


Based on the obtained equations, a graph of the dependence of the angle of separation of the component of the potato heap from the apron at different speeds was constructed.

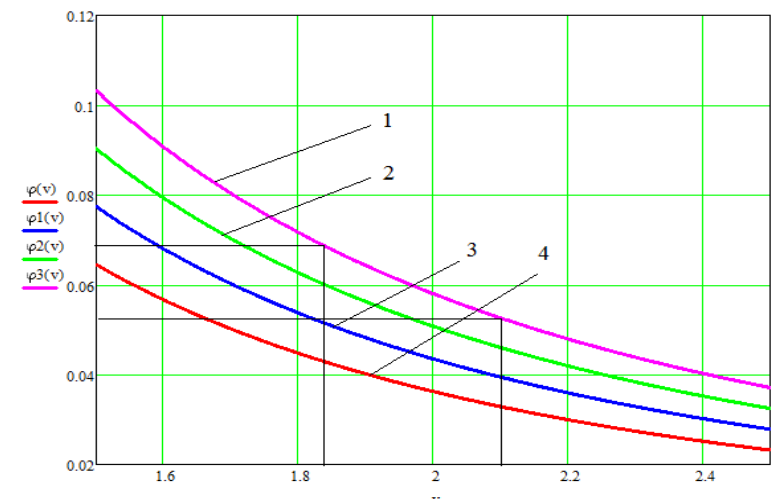

1 - roller radius $\mathrm{r}=0.08 \mathrm{~m} ; 2$ - roller radius $\mathrm{r}=0.07 \mathrm{~m} ; 3$ - roller radius $\mathrm{r}=0.06 \mathrm{~m}$; 4 - roller radius $\mathrm{r}=0.05 \mathrm{~m}$

Fig. 3. Dependence of the separation angle of the component on the speed of the elevator apron

The analysis of the graph showed that at speeds of $1.8-2.1 \mathrm{~m} / \mathrm{s}$ used on raddle chains separation angle $\varphi$ is $2.8^{\circ}-4^{\circ}$ from the position of axis $y_{1}$.In a fixed coordinate system $x 0 y$, separation angle $\varphi^{\prime}$ will be equal to $\alpha+\varphi$ and will be $22^{\circ}-24^{\circ}$.

Consider the trajectory of the components of the potato heap after tossing on the intensifier rollers. The speed of the components of the potato heap, tossed up by intensifier rollers is accepted equal to the raddle chain spee $\mathrm{d} V_{0}$. The angle of speed vector $\bar{V}_{0}$ to horizon $\varphi_{0}^{\prime}$ as the speed of the components of the heap is negligible, the air resistance is neglected. The trajectory of the components is determined, making a differential equation for the motion of the particle in the axes that coincide with the position of the raddle chain.

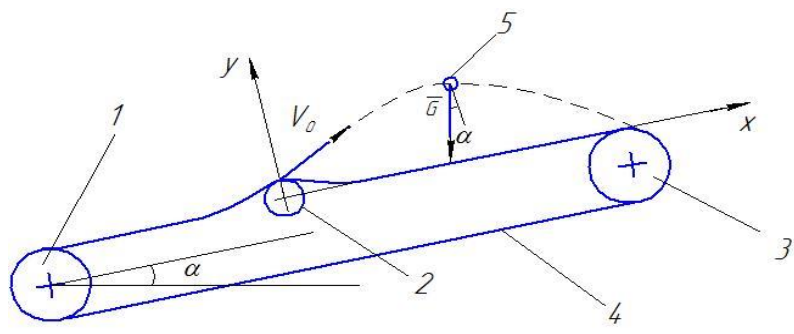

Fig. 4.Calculation scheme to determine the trajectory of a component of a potato heap

We write the differential equations

$$
\left\{\begin{array}{l}
m \frac{d^{2} x}{d t^{2}}=-G \sin \alpha \\
m \frac{d^{2} y}{d t^{2}}=-G \cos \alpha
\end{array}\right.
$$

where $m$ is the mass of the final component, $\mathrm{kg}$; $\alpha$ is the elevator tilt angle to the horizon;

$G$ is the weight of a component of a potato heap. $g$ is gravity acceleration, $\mathrm{m} / \mathrm{s}^{2}$.
Having transformed and integrated one gets

$$
\left\{\begin{array}{l}
V_{x}=V_{o x}-g t \sin \alpha \\
V_{y}=V_{o y}-g t \cos \alpha
\end{array}\right.
$$

Taking into account that

$$
V_{x}=\frac{d x}{d t}, V_{y}=\frac{d y}{d t}, V_{o x}=V_{o} \cdot \cos \left(\varphi^{\prime}\right) ; V_{o y}=V_{o} \sin \left(\varphi^{\prime}\right)
$$

one substitutes them into the expression and integrates

$$
\left\{\begin{array}{l}
x=x_{0}+V_{0} t \cos \left(\varphi^{\prime}\right)-g t^{2} \sin \alpha \\
y=y_{0}+V_{0} t \sin \left(\varphi^{\prime}\right)-g t^{2} \cos \alpha
\end{array}\right.
$$

Build the component trajectory in the program.

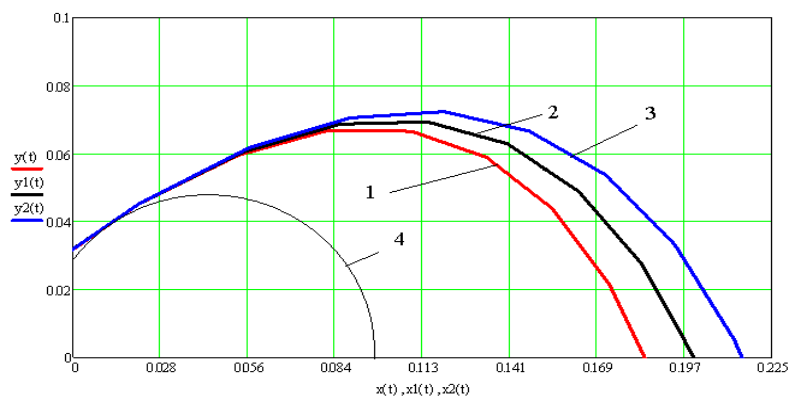

1 - the trajectory when the elevator speed of $1.9 \mathrm{~m} / \mathrm{s}$;

2 - the trajectory when the elevator speed of $2.0 \mathrm{~m} / \mathrm{s}$;

3 - the trajectory when the elevato rspeed of $2.1 \mathrm{~m} / \mathrm{s}$;

4 - the intensifier roller position

Fig. 5. Trajectory of a component of a potato heap after a jump on an intensifier

The analysis of the trajectory of the components of the potato heap shows that due to the elasticity of the combined rods, there is possibilities of intensive exposure to digged down slice of furrow. On the other hand, the use of lightweight rods will reduce the energy consumption for the drive of the separating working body.

\section{Discussion}

Researches of N.V. Byshov, S.N. Borychev, M.Yu. Kostenko, I.E. Kuschev, G.K. Rembalovich, A.A. Sorokin, G.D. Petrov, I.A. Uspenskiy and others show that reducing the power spent on the drive of the working bodies depends on their mass [1,2].

In the work of I.A. Uspenskiy [1] the technological process of a potato harvester is presented in the form of a system that considers the harvester as a set of individual working bodies and involves improving the process and reducing energy costs based on the rationale for rational operating modes.

I.E. Kuschev [1] investigated for a potato harvester the possibility of changing the technological process with disconnecting and connecting additional working bodies during soil separation. The tests of potato harvesters were based on the use of strain gauges on all power devices of the potato harvester. The essence of the method was that the power consumption of the combine was determined by key points. According to the obtained 
values, power consumption was determined in each working body or for the machine as a whole. Power consumption at this working body in idle mode was 1.58 $\mathrm{kW}$ and it was from 3.96 to $5.84 \mathrm{~kW}$ in the technological mode.

At the second elevator, the consumed power was $0.84 \mathrm{~kW}$ in the idle mode whereas in the technological mode it was from 1.85 to $2.56 \mathrm{~kW}$.

Soil separation on the main elevator is about $49 \%$. In harvesting machines, a raddle chain is the main working body that carries out separation according to geometric dimensions. It is worth noting the separation capacity of the second elevator, which amounted to $20 \%$.

These data show that the modernization of the elevator rods for the best resistance to the loads acting on it is an urgent task that will reduce the power consumption of the potato harvester.

Based on the analysis of the research results, power parameters and the separating ability of the main working bodies were generalized. The obtained values of the power consumption of the working areas are presented in Fig. 6.

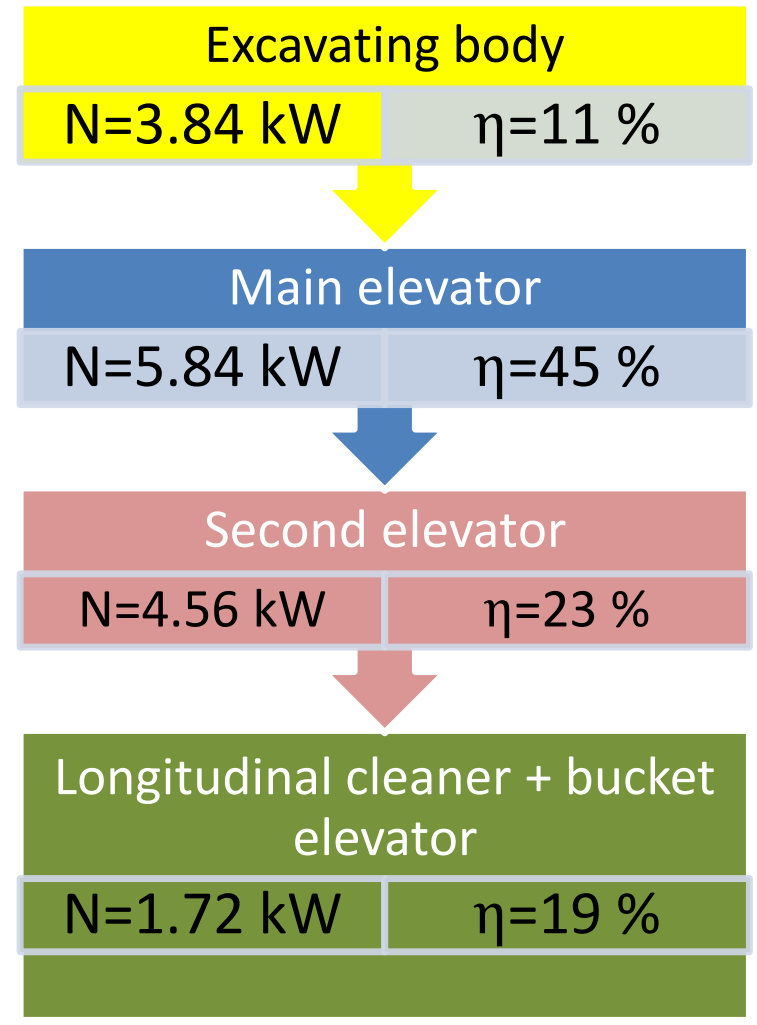

Fig. 6. Distribution scheme of power parameters and separating capacity for the main working body of a potato harvester

The feasibility study and energy assessment of existing and proposed separating working bodies made it possible to establish that when using an elevator with rods made of composite material on a root-harvesting device, the following parameters can be improved:

- increase the efficiency of tubers separation by $10-15 \%$;

- reduce damage to root crops by $9-12 \%$ [1].
An equally significant improvement will be a decrease in the mass of the working body as a whole due to the fact that composite rods are lightweight and in this component they also have an advantage over metal counterparts. The mass of one metal bar is $0.42 \mathrm{~kg}$ despite the fact that the mass of a similar composite material bar is $0.23 \mathrm{~kg}$ Analysis of the technical parameters of the working bodies is presented in Table 1 .

Table 1. Technical parameters of the working bodies

\begin{tabular}{|c|c|c|}
\hline Parameters & $\begin{array}{c}\text { Existing raddle } \\
\text { chain }\end{array}$ & $\begin{array}{c}\text { Proposed raddle } \\
\text { chain }\end{array}$ \\
\hline $\begin{array}{c}\text { The mass of the } \\
\text { working body, } \mathrm{kg}\end{array}$ & 38.1 & 24.2 \\
\hline $\begin{array}{c}\text { Specific energy } \\
\text { consumption, } \mathrm{kW} / \mathrm{t}\end{array}$ & 0.54 & 0.42 \\
\hline
\end{tabular}

Based on this, it is worth noting a significant difference in the weight of the rods, which is almost $50 \%$, a similar percentage is observed for the total mass of the working body and, in turn, reflects the energy consumption of the combine as a whole.

\section{Conclusion}

The improvement of potato harvesting machines is based on the establishment of power-loaded areas, working bodies on a potato harvester with the subsequent development of schemes for the modernization of the most loaded working bodies. The analysis of scientific works of this orientation showed that the modernization of the main elevator is an urgent task, due to the fact that this working body is the most loaded. The power consumption at the main elevator is $1.58 \mathrm{~kW}$ in the idle mode and $5.84 \mathrm{~kW}$ in the process mode. Based on the analysis of existing working developments in this area, one of the possible directions to modernize the working body has been determined and that is replacing the elevator metal bars with similar bars made of composite material, which will significantly improve separation to $86.1 \ldots 92.2 \%$ and reduce specific energy consumption $0.35 \ldots 0.47 \mathrm{~kW} / \mathrm{t}$. It is also worth noting the reduction in the cost of manufacturing a bar and the high reliability of the elevator with rods from composite material.

\section{References}

1. N.V. Byshov, S.N. Borychev, N.I. Vereschchagin et al., The technology of harvesting potatoes in difficult field conditions using promising solutions in the design and maintenance of combines, Collective monograph (RSATU, Ryazan, 2015)

2. A.J. Haverkort, B.V. Anisimov, eds., Potato production and innovation technologies, (Wageningen Academic Pablishers, The Netherlands, 2007)

3. T.V. Savchenko, A.V. Ulez ko, L.V. Kiyashchenko, A.A. Tyutyunikov, Development of the family economies of the agricultural sector of Russia, Int. Busin. Manag., 9(6), 1186-11892 (2015) 
4. H. Erdal, G. Erdal, K. Esengun, An analysis of production and price relationship for potato in Turkey: a distributed lag model application, Bulg. J. Agric. Sci., 15, 243-250 (2009)

5. Y. Wang, T.L. Brandt, N.L. Olsen, A Historical Look at Russet Burbank Potato (Solanumtuberosum L.) Quality Under Different Storage Regimes', Amer. J. of Potato Res., 93(5), 474-484 (2016)
6. K. K. Chawla, Composite Materials, 3rd ed., vol. XXIII (2012)

7. Composite Materials, Fabrication Handbook (Publications, IncPaperback, Wolfgang, 1 December 2010)

8. Kartoffelproduktion: Betriebs- und arbeitswirtschaftliche Kalkulationen, Hrsg.: Kuratorium fur Technik und Bauwesen in der Landwirtschaft (Herwart Bohm, 2011) 